\title{
Chemosensors - Welcome to a New Open Access Journal Intended to Cover All Aspects of Chemical Sensing
}

\author{
Igor L. Medintz \\ Center for Bio/Molecular Science and Engineering, Code 6900, U.S. Naval Research Laboratory, \\ 4555 Overlook Ave, SW, Washington, DC 20375, USA; E-Mail: Igor.medintz@nrl.navy.mil
}

Received: 29 November 2012 / Accepted: 6 December 2012 / Published: 10 December 2012

It gives me great pleasure to welcome you to Chemosensors, a new online-only journal established by the Multidisciplinary Digital Publishing Institute (MDPI, Basel, Switzerland) with the intent of covering all aspects of chemical sensing. The ability to sense or detect/identify and quantitate a chemical entity, and in particular accomplish this through the use of chemical means, has never been a more important part of our society. Chemosensing permeates diverse fields including healthcare (e.g., blood chemistry analysis), food safety (e.g., detecting contamination and spoilage), environmental monitoring (e.g., air and water quality), product and manufacturing assurance (e.g., purity and efficacy), household safety (e.g., smoke detection), forensics (e.g., drug analysis), and biological research (e.g., quantitating DNA or monitoring intracellular homeostasis), to name but a paltry few areas. Indeed, the application of chemosensing has become such an integrated aspect of modern society that trying to compile a comprehensive list of where it is utilized or relied on is almost impossible. Equally daunting is trying to compile a comprehensive list of all the different sensing techniques, types of analysis, modes of signal transduction, instruments and similar type aspects. The pace of new developments in this field is both remarkable and continuously accelerating with new products and applications being developed on an almost unceasing basis.

With all of the above in mind, rather than being tightly focused on just one aspect - it is our aim to give researchers a forum to publish and read about all the endeavors that relate to this discipline and it is here that I would like to elaborate a little. Beyond the normal menu of scientific papers describing experimental studies, if you would like to publish something that is normally not considered at a standard chemistry journal - then this may be just the right forum for you. Subjects that are entertained here include, but are far from limited to, results from field trials, validation and testing of a new instrument, issues to do with automation or computer code, historical retrospectives and developments in the field, integration or arraying of sensors, issues and studies relating to manufacturing and production, sensor networks, description and preliminary application of a new instrument or technique, completely theoretical papers, perspectives or outlooks on a particular topic, financial or 
implementation foci, description of potential problems, educational and training issues, results from surveys and the like, descriptions of new materials and new types of bioconjugation or modification chemistry, etc. In summary, if it is related to chemical sensing in any way - then it is appropriate and will be fairly considered by this journal.

Our editorial board reflects the multidisciplinary nature of this field and they have been selected to reflect the diversity and expertise needed. The editorial board, myself included, and the team at MDPI are committed to making this a successful journal. As such, we will focus on a rapid review and processing of all manuscripts. We will not place any size limits on manuscripts and encourage you to go into great detail in the Methodology section. This will allow the work to be more easily reproduced and adopted by others in the field. Indeed, the latter is something that is far too commonly overlooked in many published papers these days. We welcome all manner of manuscript from a short communication or perspective to extended reviews and lengthy scientific papers. If you would like to coordinate or guest edit a special issue on a particular-focused topic, please feel free to contact us and discuss your ideas. Lastly, we realize that the status and impact of this journal ultimately depends on you and the quality of manuscripts you submit. We welcome your submissions and look forward to publishing a plethora of novel, interesting, and innovative manuscripts that not only reflect the richness of science behind chemical sensing, but also help drive it in the future.

(C) 2013 by the authors; licensee MDPI, Basel, Switzerland. This article is an open access article distributed under the terms and conditions of the Creative Commons Attribution license (http://creativecommons.org/licenses/by/3.0/). 